\title{
Spherical paramagnetic contribution to shielding tensor analysis of nuclear magnetic resonance signals in gum cancer cells, tissues and tumors
}

\author{
Alireza Heidari ${ }^{1,2 *}$ and Ricardo Gobato ${ }^{3}$ \\ ${ }^{1}$ Faculty of Chemistry, California South University, 14731 Comet St. Irvine, CA 92604, USA \\ ${ }^{2}$ American International Standards Institute, Irvine, CA 3800, USA \\ ${ }^{3}$ Green Land Landscaping and Gardening, Seedling Growth Laboratory, 86130-000, Parana, Brazil
}

\begin{abstract}
In the current paper, spherical paramagnetic contribution to shielding tensor analysis of nuclear magnetic resonance signals in gum cancer cells, tissues and tumors are investigated. Owing to its high chemical specificity and non-invasive detection capability, in the last decade, spherical paramagnetic contribution to shielding tensor analysis of nuclear magnetic resonance signals in gum cancer cells, tissues and tumors has found wide application in gum cancer screening and diagnosis. In this paper, we describe recent results obtained by applying spherical paramagnetic contribution to shielding tensor analysis of nuclear magnetic resonance signals in gum cancer cells, tissues and tumors to osteosarcoma and chondrosarcoma; the two main bone tumors. The results show the remarkable potential of spherical paramagnetic contribution to shielding tensor analysis of nuclear magnetic resonance signals in gum cancer cells, tissues and tumors to discriminate between subcellular components inside osteo-differentiated osteoblasts and osteosarcoma cells or; at level of tissues; to discriminate chondrogenic tumors giving the possibility to grade the level of malignancy the cartilaginous tumors under investigation.
\end{abstract}

\section{Introduction}

Gum cancer remains the world's grand challenge. There is an urgent need for development of new techniques first for gum cancer screening, diagnosis, and then for intraoperative surgical guidance. Spherical paramagnetic contribution to shielding tensor analysis of nuclear magnetic resonance signals in gum cancer cells, tissues and tumors has long been used to assess chemical compositions in cells and tissues, based on interaction with the vibrational modes of common molecular bonds in the sample. Thus, the alteration of molecular signatures in a cell or tissue undergone disease transformation can be detected by spherical paramagnetic contribution to shielding tensor analysis of nuclear magnetic resonance signals in gum cancer cells, tissues and tumors non-invasively without labelling. It is conceivable that spherical paramagnetic contribution to shielding tensor analysis of nuclear magnetic resonance signals in gum cancer cells, tissues and tumors is a desirable tool for gum cancer diagnosis [1-10].

\section{Results and discussion}

Gum cancer remains the world's grand challenge. There is an urgent need for development of new techniques for gum cancer screening, diagnosis, and intraoperative surgical guidance. spherical paramagnetic contribution to shielding tensor analysis of nuclear magnetic resonance signals in gum cancer cells, tissues and tumors has long been used to assess chemical compositions in cells and tissues, based on interaction with the magnetic modes of common molecular bonds in the sample. Thus, the alteration of molecular signatures in a cell or tissue undergone disease transformation can be detected by spherical paramagnetic contribution to shielding tensor analysis of nuclear magnetic resonance signals in gum cancer cells, tissues and tumors non-invasively without labelling. It is conceivable that spherical paramagnetic contribution to shielding tensor analysis of nuclear magnetic resonance signals in gum cancer cells, tissues and tumors is a desirable tool for gum cancer diagnosis [1-10]. However, due to small cross section $\left(\sim 10-30 \mathrm{~cm}^{2}\right.$ per molecule), spontaneous spherical paramagnetic contribution to shielding tensor analysis of nuclear magnetic resonance signals in gum cancer cells, tissues and tumors requires a long integration time, which hinders its biological and medical applications (Figure 1).

\section{Conclusion}

Spherical paramagnetic contribution to shielding tensor analysis of nuclear magnetic resonance signals in gum cancer cells, tissues and tumors has long been used to analyze chemical compositions in biological systems. Owing to its high chemical specificity and noninvasive detection capability, spherical paramagnetic contribution to shielding tensor analysis of nuclear magnetic resonance signals in gum cancer cells, tissues and tumors has been widely employed in gum cancer screening, diagnosis, and intraoperative surgical guidance in the past ten years. In order to overcome the weak signal of spontaneous

${ }^{\star}$ Correspondence to: Alireza Heidari, Faculty of Chemistry, California South University, 14731 Comet St. Irvine, CA 92604; American International Standards Institute, Irvine, CA 3800, USA, E-mail: Scholar.Researcher.Scientist@gmail. com; Alireza.Heidari@calsu.us; Central@aisi-usa.org

Key words: spherical paramagnetic, contribution, shielding tensor analysis, nuclear magnetic resonance, signals, gum cancer cells, gum cancer tissues, tumors

Received: August 16, 2020; Accepted: August 28, 2020, Published: September 02, 2020 


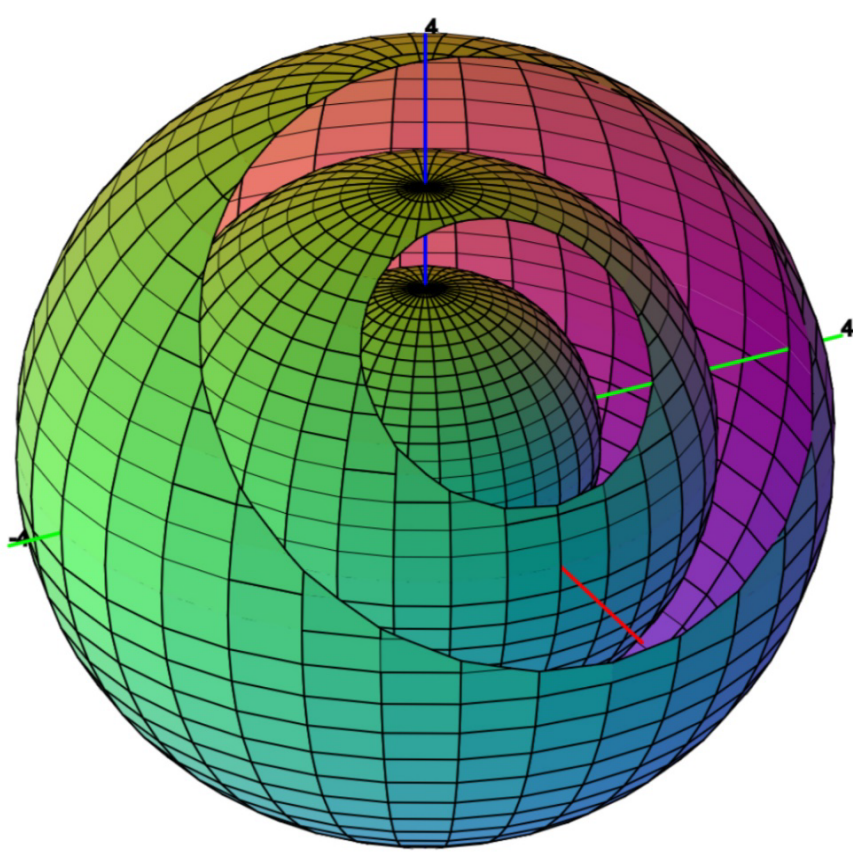

Figure 1. Spherical paramagnetic contribution to shielding tensor analysis of nuclear magnetic resonance signals in gum cancer cells, tissues and tumors

spherical paramagnetic contribution to shielding tensor analysis of nuclear magnetic resonance signals in gum cancer cells, tissues and tumors, coherent spherical paramagnetic contribution to shielding tensor analysis of nuclear magnetic resonance signals in gum cancer cells, tissues and tumors and surface-enhanced spherical paramagnetic contribution to shielding tensor analysis of nuclear magnetic resonance signals in gum cancer cells, tissues and tumors have been developed and recently applied in the field of gum cancer research. This review focuses on innovative studies of the use of spherical paramagnetic contribution to shielding tensor analysis of nuclear magnetic resonance signals in gum cancer cells, tissues and tumors in gum cancer diagnosis and their potential to transition from bench to bedside.

\section{Acknowledgments}

This study was supported by the Cancer Research Institute (CRI) Project of Scientific Instrument and Equipment Development, the National Natural Science Foundation of the United Sates, the International Joint BioSpectroscopy Core Research Laboratory Program supported by the California South University (CSU), and the Key project supported by the American International Standards Institute (AISI), Irvine, California, USA.

\section{References}

1. Heidari A, Brown C (2015) Study of composition and morphology of cadmium oxide (CdO) nanoparticles for eliminating cancer cells. J Nanomed Res 2(5): 20.

2. Heidari A, Brown C (2015) Study of surface morphological, phytochemical and structural characteristics of rhodium (III) oxide $\left(\mathrm{Rh}_{2} \mathrm{O}_{3}\right)$ nanoparticles. International Journal of Pharmacology, Phytochemistry and Ethnomedicine 1(1): 15-19.

3. Heidari A (2016) An experimental biospectroscopic study on seminal plasma in determination of semen quality for evaluation of male infertility. Int $J$ Adv Technol 7: $\mathrm{e} 007$.

4. Heidari A (2016) Extraction and preconcentration of N-Tolyl-Sulfonyl-PhosphoramidSaeure-Dichlorid as an anti-cancer drug from plants: a pharmacognosy study. $J$ Pharmacogn Nat Prod 2: e103.

5. Heidari A (2016) A thermodynamic study on hydration and dehydration of DNA and RNA-Amphiphile Complexes. J Bioeng Biomed Sci S: 006.

6. Heidari A (2016) Computational studies on molecular structures and carbonyl and ketene groups' effects of singlet and triplet energies of Azidoketene $\mathrm{O}=\mathrm{C}=\mathrm{CH}-\mathrm{NNN}$ and Isocyanatoketene $\mathrm{O}=\mathrm{C}=\mathrm{CH}-\mathrm{N}=\mathrm{C}=\mathrm{O} . J$ Appl Computat Math 5: e142.

7. Heidari A (2016) Study of irradiations to enhance the induces the dissociation of hydrogen bonds between peptide chains and transition from helix structure to random coil structure using ATR-FTIR, Raman and 'HNMR Spectroscopies. J Biomol Res Ther 5: e146.

8. Heidari A (2016) Future prospects of point fluorescence spectroscopy, fluorescence imaging and fluorescence endoscopy in photodynamic therapy (PDT) for cancer cells. J Bioanal Biomed 8: e135.

9. Heidari A (2016) A bio-spectroscopic study of DNA density and color role as determining factor for absorbed irradiation in cancer cells. Adv Cancer Prev 1: e102.

10. Heidari A (2016) Manufacturing process of solar cells using cadmium oxide (CdO) and rhodium (III) Oxide $\left(\mathrm{Rh}_{2} \mathrm{O}_{3}\right)$ nanoparticles. J Biotechnol Biomater 6: e125.

Copyright: (C2020 Heidari A. This is an open-access article distributed under the terms of the Creative Commons Attribution License, which permits unrestricted use, distribution, and reproduction in any medium, provided the original author and source are credited. 